\title{
Changes in neutrophil count, creatine kinase and muscle soreness after repeated bouts of downhill running
}

\author{
Lucille L Smith (PhD) ${ }^{1}$ \\ Stuart J Semple (D Tech) ${ }^{1}$ \\ Andrew J McKune (D Tech) ${ }^{1}$ \\ Nevel Neveling (Biokinetics/M Tech) ${ }^{1}$ \\ Miguel Caldeira (Biokinetics/B Tech) ${ }^{1}$ \\ Jean-Marie Swanepoel (B Tech) ${ }^{1}$ \\ Lebogang Tsomele (B Tech) ${ }^{1}$ \\ Melissa Naidoo (BSc Hon) ${ }^{1}$ \\ Emmanuel Sibanda (M Tech) ${ }^{2}$ \\ ${ }^{1}$ Department of Sport, Rehabilitation and Dental Sciences, Tshwane University of Technology, Pretoria \\ ${ }^{2}$ Department of Statistical Services, Tshwane University of Technology, Pretoria
}

\begin{abstract}
Objective. A primary objective was to examine circulating neutrophil count after repeated bouts of downhill running. An additional aim was to determine creatine kinase (CK) levels during the initial 12 hours, after repeated DHRs.

Design. Eleven healthy, untrained Caucasian males performed $2 \times 60$ min bouts of DHR (-13.5\%), spaced 14 days apart, at a speed equal to $75 \% \mathrm{VO}_{2 \max }$ on a level grade. Blood was collected before, after, and every hour for 12 hours, and every 24 hours for 6 days. Absolute neutrophil count, CK, and delayed-onset muscle soreness (DOMS) were assessed. Results were analysed using repeated measures ANOVA $(p<0.05)$ with appropriate post hoc tests.
\end{abstract}

Results. There were no significant differences in neutrophil count $(p=0.24)$ during the $12-h$ period following run 1 (mean \pm se, $\left.6.45 \pm 0.2910^{-9} . .^{-1}\right)$ versus run $2(5.96 \pm 0.09$ $\left.10^{-9} . .^{-1}\right)$, or during the $24-\mathrm{h}$ periods for run $1(3.48 \pm 0.09$ $\left.10^{-9} . I^{-1}\right)$ or run $2\left(3.47 \pm 0.0910^{-9} . I^{-1}\right)$. During the initial $12-\mathrm{h}$

\section{CORRESPONDENCE:}

Lucille L Smith

Department of Sport, Rehabilitation and Dental Sciences

Tshwane University of Technology

South Africa

E-mail: smithll@tut.ac.za

Tel : 012-382-5921

Cell: 082-561-8932 period, there was a significant interaction effect $(p=0.0001)$ for CK with differences between bouts seen between 3 - $12 \mathrm{~h}$; differences remained evident at $24 \mathrm{~h}$ and at 96 $144 \mathrm{~h}$. In all muscle groups, DOMS was significantly lower after run 2 compared with run 1 .

Conclusion. The lack of significance in neutrophils, as well as the early onset of difference in CK between run 1 and run 2 were attributed to the type of eccentric protocol used. It was proposed that future studies be more cognisant of whether the eccentric mode is predominantly low-intensity long-duration or high-intensity short-duration.

\section{Introduction}

An unaccustomed bout of eccentrically biased exercise results in trauma to muscle and/or connective tissue. It is now well established that if a similar bout is repeated within several days ${ }^{22}$ to several months, ${ }^{15}$ there is a significant reduction in direct and indirect markers of muscle damage. ${ }^{13}$ This response is referred to as the repeated bout effect and is believed to represent a positive training adaptation. ${ }^{13}$

Several hypotheses have been proposed in an attempt to explain this phenomenon. McHugh $^{13}$ has classified these hypotheses as the neural, mechanical, and cellular adaptation. $^{13}$ One aspect of the cellular adaptation hypothesis that is gaining in acceptance is related to the initial acute inflammatory response after the first bout of eccentrics. ${ }^{16}$ Although acute inflammation promotes healing, an undesirable 'side-effect' of this process involves the release of catabolic substances that inadvertently degrade surrounding healthy tissue. Pizza and colleagues ${ }^{18,19}$ have suggested that the repeated bout effect may be related to 
an attenuated inflammatory response after the second bout. This suggestion has been supported by the observation that there is a significantly reduced number of circulating neutrophils after the second bout of eccentrics, compared with the first. Since neutrophils are typically the first white blood cells to enter damaged tissue and are instrumental in initiating an acute inflammatory response they reasoned that reduced circulating numbers of neutrophils after bout two, would result in reduced infiltration into damaged tissue and a reduction in subsequent inflammatory events.

In contrast to the findings of reduced neutrophilia after bout $2^{, 18,19}$ a previous study ${ }^{21}$ found no significant differences in neutrophil count when a second bout of eccentrically biased exercise was performed a few weeks after the initial bout. However, a primary difference between the two studies was the exercise protocol. Pizza et al. ${ }^{18,19}$ used highintensity, low-volume resistance-like eccentric contractions of the elbow flexors, while the aforementioned study ${ }^{21}$ used repeated bouts of downhill running, which incorporated lowintensity high-volume aerobic-like eccentric contractions. An alternate reason for the lack of consensus between these studies could have been that in the previous downhill running study, $^{21}$ the intensity, the steepness of the gradient and the duration of the running bouts were not sufficiently strenuous. Therefore, a primary purpose of the present study was to reexamine circulating white cell counts, specifically neutrophils and also monocytes after two bouts of downhill running, using a higher intensity and longer duration.

Serum creatine kinase (CK) has frequently been used as an indirect marker of muscle damage. With regard to repeated bouts of eccentrics, CK has consistently been shown to be significantly lower after the second bout of eccentrics. $3,8,14,18,21$ However, in previous downhill running studies ${ }^{3,5,21}$ CK was only measured at 24-h intervals; whether differences in serum CK are evident at an earlier time point after downhill running has not been investigated. Thus, an additional purpose of this study was to assess total circulating $\mathrm{CK}$ at 3-h intervals during the initial $12 \mathrm{~h}$, as well as at 24-h intervals for $6 \mathrm{~d}$, following both downhill runs.

\section{Methods}

\section{Subject selection}

Eleven healthy, active but untrained Caucasian males were recruited for the study; 'untrained' was defined as not having engaged in regular sport or physical activity for at least 6 months. Selection criteria for subjects included the following: age between 18 and 30 years; no history of leg injury or any other medical condition that would be exacerbated by two bouts of downhill running; no regular usage of any antiinflammatory medication.

\section{Initial screening}

The individuals selected to participate were scheduled for screening in the Exercise Testing Laboratory (ETL). During the first visit they were required to read and sign an informed consent previously approved by the University Ethics Com- mittee and in accord with guidelines established by the American College of Sports Medicine. Height and body mass were assessed. Body composition was assessed using a 7-site skinfold caliper (Harpenden, British Instruments, London). The sites were: chest, midaxillary, triceps, subscapular, abdomen, supra-iliac, thigh). The equation used was: body density $=1.112-0.00043499$ (sum of 7 skinfolds) +0.00000055

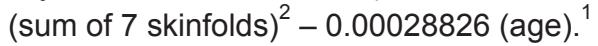

\section{Assessment of $\mathrm{VO}_{2 \max }$ and determination of individual running speeds}

Subjects were instructed to eat a light meal 3 hours prior to the $\mathrm{VO}_{2 \max }$ testing. To determine $\mathrm{VO}_{2 \max }$, subjects were required to walk/run on a treadmill using the Bruce protocol. ${ }^{1}$ The test was performed on a Quinton 90 treadmill (Quinton Instrument Co. Seattle, Washington). Continuous respiratory measurements were recorded by means of the MedGraphics $\mathrm{CardiO}_{2}$ combined $\mathrm{VO}_{2} / \mathrm{ECG}$ exercise system (Medical Graphics Corporation Chicago, Illinois). Throughout the test, heart rate was recorded at the end of each minute using a Polar ${ }^{\mathrm{TM}}$ Heart Rate Monitor (Accurex 2, Polar Electro, Finland). Ratings of perceived exertion (RPE) were recorded at the end of each stage (every 3 minutes), as well as when subjects reached volitional exhaustion; the 15-point Category Scale was used. ${ }^{1}$ The test was accepted as $\mathrm{VO}_{2 \max }$ if two of the following criteria were attained: RER > 1.1 and/or, RPE $\geq$ than 19 on the 15-point RPE Scale, and/or maximum heart rate $(\mathrm{HR})$ within \pm 20 beats of age predicted HRmax.

After the test, $\mathrm{VO}_{2 \max }\left(\mathrm{ml} \mathrm{kg}^{-1} \cdot \mathrm{min}^{-1}\right)$ values were converted into metabolic equivalents (METS). Seventy-five per cent of the peak MET capacity was then calculated. Metabolic equations were used to determine the speed on a level grade that would elicit this MET capacity. ${ }^{1}$ This treadmill speed was the designated speed for each subject for both downhill runs.

\section{Eccentrically biased downhill run}

During the $72 \mathrm{~h}$ prior to the downhill run, subjects were instructed to ingest a normal mixed diet, to be well hydrated, and to refrain from any strenuous exercise. Each subject performed two identical bouts of downhill running spaced 14 days apart (run 1 and run 2). On both days subjects ran between 5:00 am and 11:00 am and repeated runs were at approximately the same time for each subject. Subjects were instructed to fast overnight, but were encouraged to drink ad libitum, to ensure euhydration. At the start of both runs, subjects warmed up for 5 minutes by running on a level grade at the pre-determined speed. The treadmill was then lowered to $-13.5 \%$ and subjects ran for $60 \mathrm{~min}$. Heart rate was recorded at the end of each minute (Polar ${ }^{\mathrm{TM}}$ Heart Rate Monitor, Accurex 2, Finland).

\section{Blood sampling}

On arriving in the laboratory, before run 1 and run 2, subjects were required to sit quietly for $10 \mathrm{~min}$. A qualified phlebotomist then inserted a venous catheter (22 gauge, $2.2 \mathrm{~cm}$ ), 
which was kept patent for the following $12 \mathrm{~h}$, using a saline solution. Blood was drawn at the following times: pre-exercise, immediately after (post), 1, 2, 3, 4, 5, 6, 7, 8, 9, 10, 11 and $12 \mathrm{~h}$ after (14 samples $\times 15 \mathrm{ml}$ per sample $=210 \mathrm{ml}$ blood over approximately $14 \mathrm{~h}$ ). In addition, subjects were required to return to the ETL 24, 48, 72, 96, 120 and $144 \mathrm{~h}$ later, for additional blood draws (6 days $\times 15 \mathrm{ml}=90 \mathrm{ml}$ ). At these times, a standard venipuncture was performed using an antecubital vein.

Plasma (5 $\mathrm{ml}$ whole blood) was collected in EDTA tubes and used for assessment of total and differential white cell count and for assessment of CK.

Serum (10 $\mathrm{ml}$ whole blood) was collected in serum separator tubes, allowed to stand at room temperature for $30 \mathrm{~min}$ and then spun down for $10 \mathrm{~min}$ at $2000 \times \mathrm{g}$. Aliquots were frozen at $-70^{\circ} \mathrm{C}$ in $0.5 \mathrm{ml}$ eppendorf tubes.

Subjects remained in the ETL for $12 \mathrm{~h}$ after run 1 and run 2. They were provided with food and fluid and encouraged to eat, and especially to drink, ad libitum.

\section{Total and differential white cell count}

Values were assessed before and immediately after exercise, and then every hour for $12 \mathrm{~h}$, as well as 24 - $144 \mathrm{~h}$. Total and differential white cell count were determined using a Cell-Dyn 3000 (Abott Laboratories, Mountain View, CA) and analysed by laser-based flow cytometry. All samples were analysed within $16 \mathrm{~h}$ of collection.

\section{Assessment of CK}

CK was assessed at the following times: before, immediately after, 3, 6, 9, 12, 24, 48, 72, 96, 120 and $144 \mathrm{~h}$ after exercise. Blood CK concentrations were determined using a Refletron blood analyser, which uses a colorometric assay procedure (Boehringer Mannheim $\mathrm{GmbH}$, Germany). EDTA blood $(32 \mu \mathrm{l})$ was pipetted onto a Refletron CK strip (Roche Diagnostics, Indianapolis, IN). The strip was inserted into the Refletron analyser for $30 \mathrm{sec}$. Printed results were recorded. If values were $>1500 \mathrm{IU} / \mathrm{I}$ the sample was diluted with equal parts distilled water; $32 \mu \mathrm{l}$ of this mixture was then pipetted onto a strip and rerun immediately. Values of diluted blood were then doubled.

\section{Assessment of DOMS}

Subjective soreness ratings were assessed before exercise and at the following times after exercise: 6, 12, 24, 48, 72, 96,120 , and $144 \mathrm{~h}$. Subjects were asked to gently palpate and move various muscle groups through a comfortable range of motion. They were then asked to rate each muscle group individually, by placing an ' $X$ ' along a visual analogue scale (VAS) of $10 \mathrm{~cm}$. The verbal anchors on this scale were ' 1 = normal' and '10 = very, very sore'. ${ }^{19}$ The distance in centimeters from the beginning of the scale to their mark was measured and this represented the muscle soreness score for that particular muscle group.

\section{Statistical analysis}

All dependent variables were analysed using a repeated measures ANOVA. Separate analyses were performed for the initial 12-h periods and for the 24-h periods with all values being compared with pre-exercise levels. Significance was set at $p \leq 0.05$. Where significant main effects or interaction effects were found, a post hoc Least Square Means was used. All values reported are means \pm se.

\section{Results}

\section{Physical characteristics of subjects}

Physical characteristics of 11 subjects were: age (years) = 19.7 \pm 0.4 ; height $(m)=1.79 \pm 0.3$; mass $(\mathrm{kg})=78.5 \pm 3.0$; body fat $(\%)=14.6 \pm 3.2 ; \mathrm{VO}_{2 \max }\left(\mathrm{ml} \cdot \mathrm{kg}^{-1} \cdot \mathrm{min}^{-1}\right)=47.8 \pm 3.6$.

The main effects in this study were bout (run 1 versus run 2) and time (initial 12-h period and then 24-h intervals for 6 d). Any interaction effects represented differences between bouts (run 1 versus run 2) at a specific time(s). Values reported are means \pm se.

\section{White cell counts}

\section{Neutrophil count (Tables I and II)}

For the initial 12-h period there was no significant bout $(p=0.24)$ or interaction $(p=0.4)$ effect. There was a significant time effect $(p=0.0001)$. Neutrophils were significantly elevated over baseline $\left(3.30 \pm 0.76,10^{-9} . .^{-1}\right)$ at $2 \mathrm{~h}(7.74 \pm 0.76$, $\left.10^{-9} . I^{-1}\right), 3 \mathrm{~h}\left(7.39 \pm 0.76,10^{-9} . \mathrm{I}^{-1}\right)$ and $4 \mathrm{~h}(9.69 \pm 0.78$, $\left.10^{-9} \cdot .^{-1}\right)$. For the 24-h comparisons there were no significant run $(p=0.9)$, time $(p=0.9)$, or interaction $(p=0.6)$ effects.

\section{Monocyte count}

For the initial $12-\mathrm{h}$ period there was no bout $(p=0.06)$ or interaction $(p=0.89)$ effect. However, there was a significant time effect $(p=0.0001)$. From $4 \mathrm{~h}$ after exercise through $12 \mathrm{~h}$, monocytes were significantly elevated over pre-exercise baseline levels $\left(0.51 \pm 0.03,10^{-9} . .^{-1}\right)$, with peak values seen at $6 \mathrm{~h}\left(0.89 \pm 0.0310^{-9} \cdot \mathrm{I}^{-1}\right)$. For the 24-h comparisons there were no significant bout $(p=0.12)$ time $(p=0.62)$ or interaction $(p=0.93)$ effects.

\section{Creatine kinase (Fig. 1)}

For the initial 12-h period there was a significant interaction effect $(p=0.0001)$. Post hoc testing revealed that values for run 1 were significantly higher than for run 2 at the following times: 3 h $\left(557 \pm 48\right.$ v. $289 \pm 48$ IU. $\left.^{-1}\right), 6$ h $(936 \pm 51$ v. $402 \pm 48$ IU. $\left.I^{-1}\right), 9$ h $\left(1242 \pm 48\right.$ v. $514 \pm 48$ IU. $\left.^{-1}\right)$ and 12 h $(1243 \pm 58$ v. $\left.535 \pm 48 \mathrm{IU} . \mathrm{I}^{-1}\right)$. There was also a significant bout effect $(p=0.0001)$, with values for run $1 \mathrm{v}$. run 2 being $727 \pm 21 \mathrm{IU}^{-1}$ v. $347 \pm 20 \mathrm{IU} . \mathrm{I}^{-1}$, respectively.

For the 24-h comparisons there was a significant interaction $(p=0.0001)$ effect. Post hoc testing revealed significant differences between run 1 and run 2, respectively, at $24 \mathrm{~h}\left(827 \pm 49\right.$ v. $\left.319 \pm 49 \mathrm{IU}^{-1} \mathrm{I}^{-1}\right), 96 \mathrm{~h}(533 \pm 49$ v. $132 \pm 49$ IU.I-1), 120 h $\left(499 \pm 49\right.$ v. $141 \pm 49$ IU. $\left.^{-1}\right)$ and 144 h $(404 \pm 49$ 
Table I. Neutrophil and monocyte count before and after repeated bouts (run 1 and run 2) of downhill running

\begin{tabular}{|c|c|c|c|c|}
\hline \multirow[b]{2}{*}{ Time (h) } & \multicolumn{2}{|c|}{ Neutrophil count $\left(10^{-9} \cdot .^{-1}\right)$} & \multicolumn{2}{|c|}{ Monocyte count $\left(10^{-9} \cdot .^{-1}\right)$} \\
\hline & Run 1 & Run 2 & Run 1 & Run 2 \\
\hline Before & $3.209 \pm 1.073$ & $3.400 \pm 1.073$ & $0.500 \pm 0.033$ & $0.509 \pm 0.033$ \\
\hline After & $4.418 \pm 1.073$ & $4.856 \pm 1.073$ & $0.463 \pm 0.033$ & $0.491 \pm 0.033$ \\
\hline 1 & $6.909 \pm 1.073$ & $6.368 \pm 1.073$ & $0.518 \pm 0.033$ & $0.509 \pm 0.033$ \\
\hline 2 & $7.957 \pm 1.073$ & $7.526 \pm 1.073$ & $0.645 \pm 0.033$ & $0.663 \pm 0.033$ \\
\hline 3 & $7.636 \pm 1.073$ & $7.139 \pm 1.073$ & $0.654 \pm 0.033$ & $0.636 \pm 0.033$ \\
\hline 4 & $7.405 \pm 1.130$ & $6.624 \pm 1.073$ & $0.731 \pm 0.035$ & $0.727 \pm 0.033$ \\
\hline 5 & $7.265 \pm 1.072$ & $6.109 \pm 1.073$ & $0.808 \pm 0.033$ & $0.800 \pm 0.033$ \\
\hline 6 & $6.979 \pm 1.130$ & $5.809 \pm 1.073$ & $0.931 \pm 0.035$ & $0.845 \pm 0.033$ \\
\hline 7 & $6.773 \pm 1.073$ & $5.527 \pm 1.073$ & $0.860 \pm 0.033$ & $0.854 \pm 0.033$ \\
\hline 8 & $6.702 \pm 1.130$ & $5.245 \pm 1.073$ & $0.841 \pm 0.035$ & $0.782 \pm 0.033$ \\
\hline 9 & $6.627 \pm 1.073$ & $5.082 \pm 1.073$ & $0.809 \pm 0.033$ & $0.727 \pm 0.033$ \\
\hline 10 & $6.396 \pm 1.129$ & $4.927 \pm 1.073$ & $0.733 \pm 0.035$ & $0.709 \pm 0.033$ \\
\hline 11 & $6.182 \pm 1.130$ & $4.791 \pm 1.073$ & $0.778 \pm 0.035$ & $0.718 \pm 0.033$ \\
\hline 12 & $5.809 \pm 1.072$ & $4.682 \pm 1.073$ & $0.718 \pm 0.033$ & $0.672 \pm 0.033$ \\
\hline
\end{tabular}

Table II. Neutrophil and monocyte count before and after repeated bouts (run 1 and run 2) of downhill running

\begin{tabular}{|c|c|c|c|c|}
\hline \multirow[b]{2}{*}{ Time (h) } & \multicolumn{2}{|c|}{ Neutrophil count $\left(10^{-9} . .^{-1}\right)$} & \multicolumn{2}{|c|}{ Monocyte count $\left(10^{-9} . .^{-1}\right)$} \\
\hline & Run 1 & Run 2 & Run1 & Run 2 \\
\hline Before & $3.209 \pm 0.224$ & $3.400 \pm 0.224$ & $0.500 \pm 0.027$ & $0.509 \pm 0.272$ \\
\hline 24 & $3.818 \pm 0.224$ & $3.282 \pm 0.224$ & $0.500 \pm 0.027$ & $0.445 \pm 0.027$ \\
\hline 48 & $3.418 \pm 0.224$ & $3.456 \pm 0.237$ & $0.463 \pm 0.027$ & $0.435 \pm 0.029$ \\
\hline 72 & $3.309 \pm 0.224$ & $3.636 \pm 0.237$ & $0.473 \pm 0.027$ & $0.465 \pm 0.029$ \\
\hline 96 & $3.436 \pm 0.224$ & $3.354 \pm 0.224$ & $0.482 \pm 0.027$ & $0.454 \pm 0.027$ \\
\hline 120 & $3.658 \pm 0.237$ & $3.518 \pm 0.224$ & $0.464 \pm 0.029$ & $0.464 \pm 0.027$ \\
\hline 144 & $3.497 \pm 0.224$ & $3.639 \pm 0.224$ & $0.484 \pm 0.027$ & $0.453 \pm 0.027$ \\
\hline
\end{tabular}

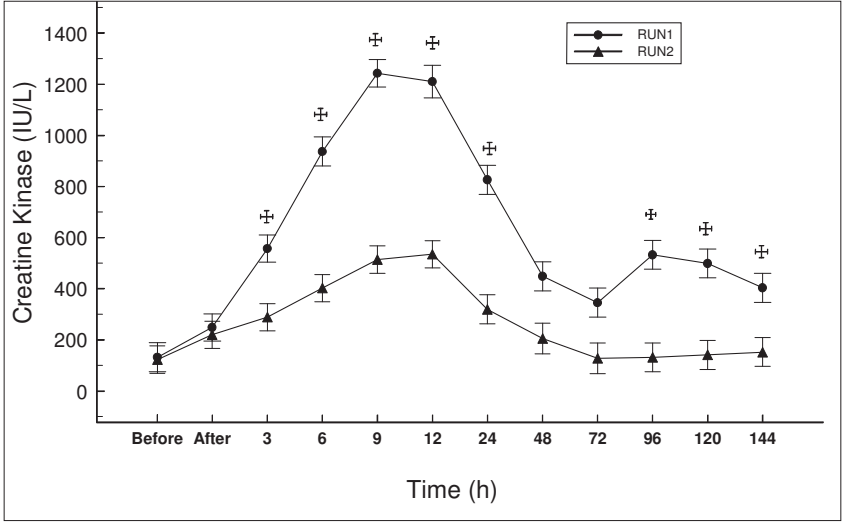

Fig. 1. Changes in blood levels of creatine kinase across time (before exercise through 144 h), for run 1 (circle) and run 2 (triangle). The cross symbol represents significant differences between bouts at specific times.

v. $\left.153 \pm 49 \mid \mathrm{IU} \cdot \mathrm{I}^{-1}\right)$. There was also a significant bout effect $(p=0.0001)$. Values for run $1\left(456 \pm 9 \mathrm{IU}^{-1} \mathrm{I}^{-1}\right)$ were significantly higher than for run $2\left(174 \pm 19 \mathrm{IU} . \mathrm{I}^{-1}\right)$.
It was interesting to note that CK peaked at $9 \mathrm{~h}$ and $12 \mathrm{~h}$ after run 1 and run 2 .

\section{Delayed-onset nuscle soreness (DOMS)}

DOMS was reported in several different muscle groups which include the following:

Upper back (Fig. 2A): There was a significant bout effect $(p=0.0004)$, with run 1 being significantly higher $(2.4 \pm 0.1)$ than run $2(1.8 \pm 0.1)$. Peak soreness for upper back occurred at $24 \mathrm{~h}$ for both runs $(4.5 \pm 0.4$ v. $2.6 \pm 0.4)$.

Lower back (Fig. 2B): There was a significant bout effect $(p=0.0001)$, with run $1(2.4 \pm 0.1)$ being significantly higher than run $2(1.6 \pm 0.1)$. Peak soreness occurred at $24 \mathrm{~h}$ for run $1(3.5 \pm 0.3)$ and run $2(1.8 \pm 0.3)$.

M. gluteus max (Fig. 2C): There was a significant bout effect $(p=0.0001)$, with run $1(2.7 \pm 0.1)$ being significantly higher than run $2(1.8 \pm 0.1)$. Peak soreness occurred at $24 \mathrm{~h}$ for run $1(3.8 \pm 0.4)$ and run $2(3.1 \pm 0.4)$.

Quadriceps (Fig. 3A): There was a significant bout $(p=0.0001)$ effect with values for run $1(3.1 \pm 0.1)$ being 


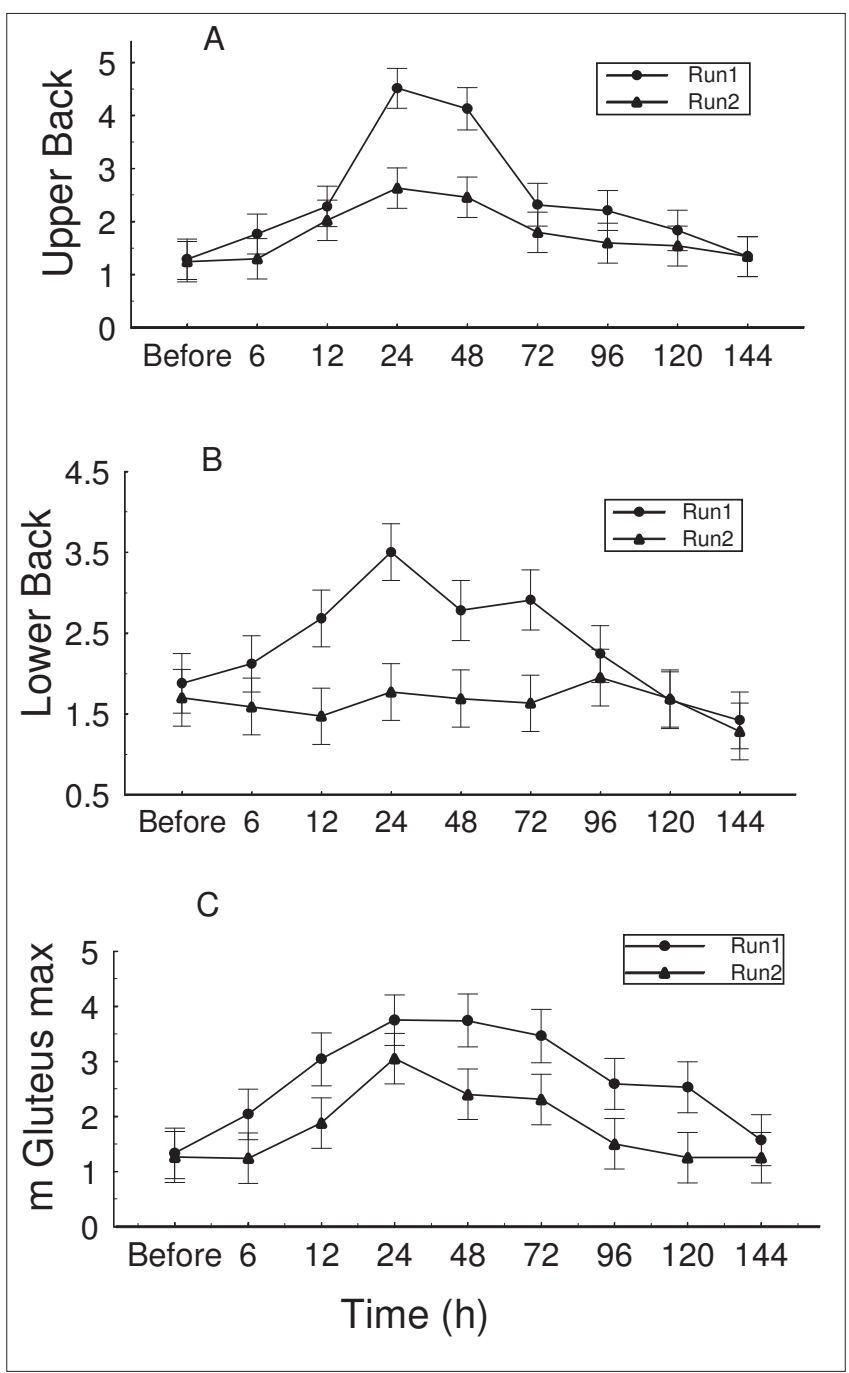

Fig. 2. This represents changes in delayed-onset muscle soreness across time for (A) upper back and (B) lower back and (C) gluteus maximus. In all muscle groups there was a significant bout effect $(p<0.05$, not shown on the graph), with values for run 2 being consistently lower than for run 1. (1 = 'normal' and 10 = 'very, very sore').

significantly higher than for run $2(1.6 \pm 0.1)$. There was also an interaction effect ( $p=0.0079$ ), with run 1 being significantly higher than run 2 at 24 (5.0 v. 2.5) 48 (4.8 v. 2.2) and $72 \mathrm{~h}$ (4.3 v. 2.1). Peak soreness occurred at $24 \mathrm{~h}$ for both runs.

Hamstrings (Fig. 3B): There was a significant bout effect $(p=0.0001)$. With run $1(2.4 \pm 0.1)$ being higher than run 2 $(1.6 \pm 0.1)$. Peak soreness occurred at $48 \mathrm{~h}$ for run $1(3.8 \pm 0.4)$ and at $24 \mathrm{~h}$ for run $2(2.3 \pm 0.3)$.

M. tibialis anterior (Fig. $3 C$ ): There was a significant bout effect $(p=0.0001)$, with run $1(2.3 \pm 0.1)$ being higher than run $2(1.5 \pm 0.1)$. Peak soreness in this muscle group occurred at $72 \mathrm{~h}$ for run $1(2.9 \pm 0.3)$ and at $48 \mathrm{~h}$ for run $2(2.0 \pm 0.3)$.

M. triceps surae (Fig. 3D): There was a significant bout effect $(p=0.0001)$ with run $1(3.3 \pm 0.2)$ significantly higher than run $2(1.9 \pm 0.2)$. Peak soreness occurred at $72 \mathrm{~h}$ for run $1(4.4 \pm 0.5)$ and at $24 \mathrm{~h}$ and $48 \mathrm{~h}$ for run $2(2.7 \pm 0.5)$

\section{Discussion}

A primary finding of this study was the confirmation of results from a previous study, ${ }^{21}$ that there were no significant differences in neutrophil count when comparing an initial bout with a subsequent bout of downhill running. This is contrary to previous findings after repeated bouts of high-intensity eccentrics, ${ }^{17,18}$ where significantly lower levels of circulating neutrophils were found after the second bout. Regarding CK, significant differences between bouts were seen at 3 , 6, 912 and $24 \mathrm{~h}$ after both bouts, as well at 96, 120 and $144 \mathrm{~h}$ after exercise. We believe that this is the first study to report this disparity in CK so soon after bouts of downhill running. Concerning DOMS, after run 1 , it was interesting to note that soreness peaked at different times in different muscle groups. Furthermore, peak soreness did not always occur at the same time points in the same muscle groups after run 1 and run 2 .

\section{Neutrophil and monocyte count}

Neutrophilia is an important aspect of acute inflammation and has been reported to occur after an initial bout of unaccustomed eccentrics. ${ }^{16}$ A critical aspect of neutrophilia is that the increased circulating count precedes the activation and migration of neutrophils into damaged tissue. ${ }^{17}$ In addition, the subsequent presence of neutrophils in damaged tissue is an initiating event in the acute inflammatory response. ${ }^{16}$ Pizza et al. ${ }^{17,18}$ have proposed that the reduced neutrophilia as well as the reduced activation of these cells after a repeated bout of eccentrics could, in part, be responsible for the repeated bout effect. They suggested that dampening of an initiating event could reduce the up-regulation of subsequent associated events.

If the above supposition is correct, a central question is why the disparity in results between various studies, which for the most part confirm a repeated bout effect? ${ }^{17,18} \mathrm{~A}$ noticeable difference between previous studies conducted by Pizza et al. ${ }^{17,18}$ and Smith et al., ${ }^{21}$ as well as in the present study, is the mode of eccentrically biased exercise used to induce muscle trauma. Pizza et al. ${ }^{17,18}$ had subjects perform two bouts of forced lengthening contractions of the forearm flexors of the non-dominant arm, separated by $3,^{18}$ or 4 weeks; ${ }^{17}$ it is proposed that this be regarded as highintensity, low-repetitions resistance-like eccentrics. On the other hand, in the present study and in a previous study, ${ }^{21}$ the mode of exercise involved downhill running; it is proposed that this be regarded as low intensity, high repetitions aerobic-like eccentrics. ${ }^{11,12,21}$ Although much emphasis has been placed on differences in training adaptations associated with high-force resistance-like exercise, ${ }^{14}$ versus low-force, high-repetitions, such as occurs in aerobic-like exercise, surprisingly, these differences have been virtually ignored in terms of exercise-induced muscle damage.

During high-force eccentrics it is possible that the primary damage is within the muscle fibre per se, ${ }^{10,14}$ since there is most likely actin and myosin cross-bridging throughout 


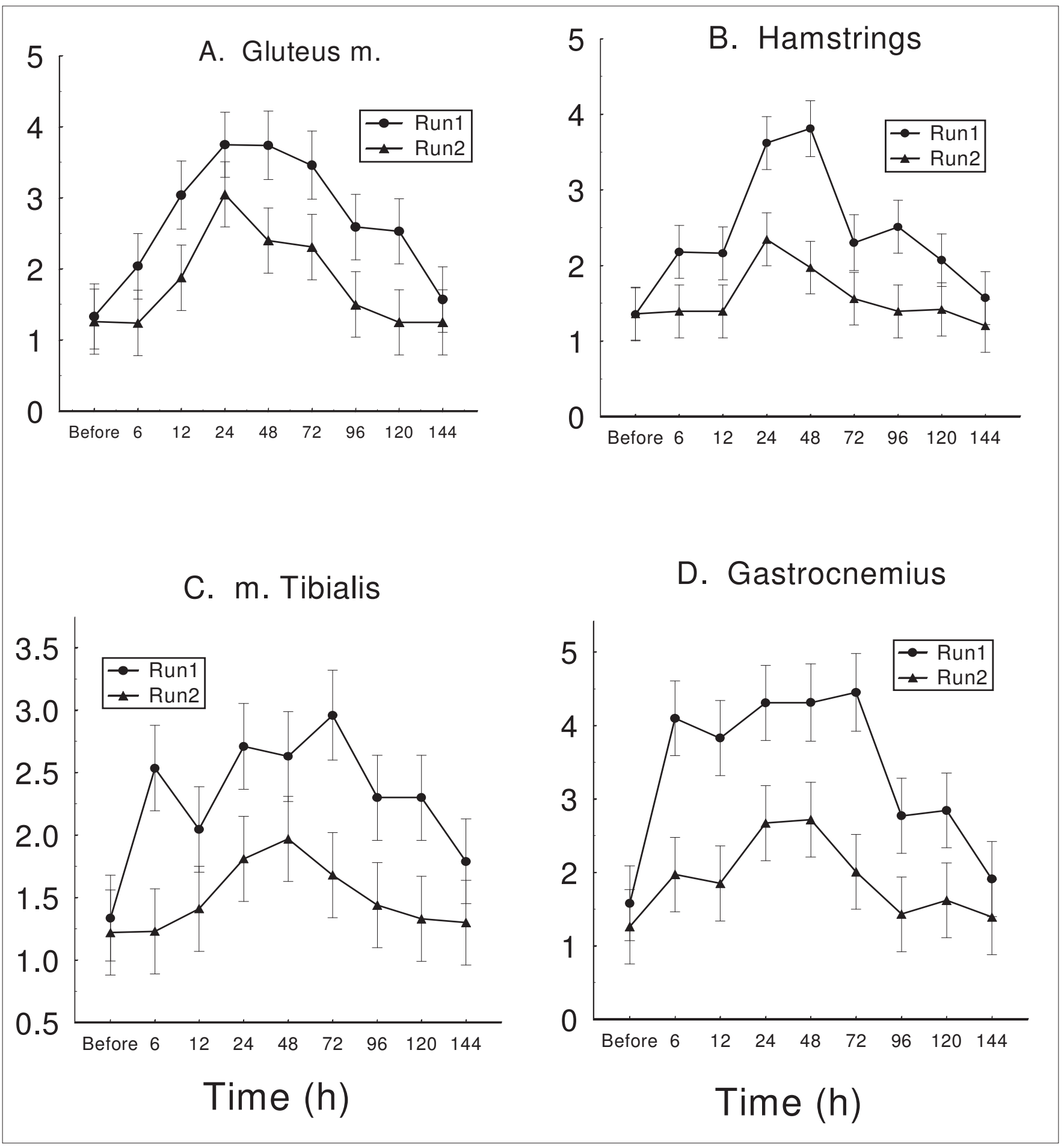

Fig. 3. These represent changes in delayed-onset muscle soreness across time for (A) quadriceps (cross symbol represents significant differences between run 1 and run 2 at each time), (B) hamstrings, (C) tibialis, (D) gastrocnemius. In all four areas, there was a significant bout effect $(p<0.05$, not shown on the graph) with values for run 2 being consistently lower than for run 1. (1 = 'normal' and 10 = 'very, very sore').

the range of motion during the lengthening contraction. However, during low-intensity high-repetition aerobiclike eccentrics, such as downhill running, ${ }^{6}$ it is proposed that most of the external load is applied at the end of the movement, producing a braking/shock absorbing function during the foot strike, with low resistance and minimal actin and myosin cross-bridging as the muscles (e.g. quadriceps) move through the swing-lengthening phase of the running motion. If the primary force is only applied at the end of the action, it is possible that the primary stress is on collagen and tendon structures outside the muscle fibre; ${ }^{10}$ this would be supported by findings of Malm and colleagues, ${ }^{11,12}$ who 
suggest that after downhill running, inflammatory factors are present in muscle epimysium and that the focal injury does not exist in muscle.

It is proposed that during high-force resistance-like eccentrics the primary damage is to the muscle fibre, while during low-force aerobic-like eccentrics the primary damage is to structures outside the muscle fibre, such as collagen and tendons. ${ }^{10}$ So differences in mode of exercise could induce differences in the target of injury. ${ }^{5}$ Whether this could induce differences in aspects of inflammation, such as differences in neutrophilia, is currently unknown.

An alternate explanation for the lack of reduced neutrophil count in the present study, after run 2, could be that the body responds differently to a focal injury (biceps trauma) versus a more diffuse injury (stress to several major muscle groups such as quadriceps and gluteal muscle). Undoubtedly the internal neuro-endocrine milieu is vastly different during these different forms of exercise/injury, and the role this may play in neutrophilia is currently not clear.

Although the focus has been on neutrophilia, monocytes/ macrophages form the second line of defense in acute inflammation. Many monocytes/macrophages are resident in tissue $\left(E D 2^{+}\right)$, while many migrate from the circulation $\left(E D 1^{+}\right)$. In the present study there were no differences in monocyte count between the two runs. However, there was a significant time effect with blood monocytes being elevated over baseline levels between 4 and $12 \mathrm{~h}$ after both bouts of exercise; these changes were remarkably similar to what has previously been reported. ${ }^{21}$ The principle that increased numbers of circulating cells precede migration to injured tissue would be in keeping with the notion that there is a similar infiltration of monocytes after both bouts of eccentrics. ${ }^{9}$

\section{Creatine kinase}

Although CK does not correlate with histological evidence of skeletal muscle damage ${ }^{23}$ it is still consistently used as an indirect marker of muscle damage. ${ }^{6,24}$ Several notable observations were seen in the present study, in response to the downhill running.

An interesting finding was related to significant differences between run 1 and run 2 starting at $3 \mathrm{~h}$ after the downhill running. Previous studies, using downhill running, have generally taken blood samples starting at $24 \mathrm{~h}$ after the exercise bout. $^{2,3,12}$ We believe this is the first study to demonstrate that significant differences occur as early as $3 \mathrm{~h}$ after the downhill run.

In the present study CK levels peaked between 9 and $12 \mathrm{~h}$ after both bouts. This early difference in CK seen after downhill running contrasts with differences reported after high-intensity resistance-like exercise (maximal exercise of elbow flexors). ${ }^{6}$ After repeated bouts of elbow flexors, significant differences between $\mathrm{CK}$ levels after bout 1 and 2 are generally seen at a later time period ${ }^{6}$ such as at 12 and $24 \mathrm{~h}^{18}$ or at 3 and $4 \mathrm{~d}^{8}$

It is suggested that the significant differences in CK, in the present study, seen initially at $3 \mathrm{~h}$ after run 1 and run 2 , as well as the time for peak values ( $9-12 \mathrm{~h}$ after run 1$)$, were due to the mode of exercise. Downhill running undoubtedly increases metabolic rate, as is evidenced by increased heart rates and oxygen consumption, ${ }^{6}$ both during and after the exercise, compared with exercise that involves high intensity/ low repetitions and that induces more focal injury. The increased metabolic rate could have resulted in a more rapid efflux of creatine kinase from the damaged tissue into the circulation. $^{16}$

In the present study, the overall finding of significantly lower CK values after run 2 concurs with previous studies on the repeated bout effect. However, when comparing peak CK values between the different types of eccentric protocols, values are consistently lower for the downhill running protocols. ${ }^{6}$ The peak mean CK seen in the present study was approximately $1200 \mathrm{IU}^{-1} \mathrm{I}^{-1}$ compared with a mean peak CK

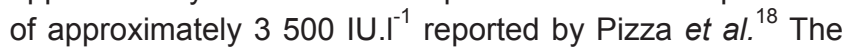
idea of lower CK levels being associated with eccentrically biased downhill running ${ }^{6}$ would support the idea that there is less damage to the muscle fibers per se (as was suggested above) and possibly more damage focused on the extramuscle collagen and tendon structures. ${ }^{4}$

An additional observation is related to changes in $\mathrm{CK}$ over the entire period (before exercise through $144 \mathrm{~h}$ after). As stated earlier, there was an initial peak between 9 and 12 $\mathrm{h}$ and then a secondary reduced, but significant peak at 96 and $120 \mathrm{~h}$. This biphasic pattern is similar to that reported by Schwane and Armstrong, ${ }^{20}$ in which rodents ran downhill on a treadmill, and is also similar to what was previously reported after downhill running in human subjects. ${ }^{21}$ Although speculative, it is suggested here that the initial peak reflects the primary mechanical injury, while the secondary peak reflects the secondary metabolic/biochemical injury. ${ }^{7,14}$

\section{Delayed-onset muscle soreness}

DOMS was assessed in 7 different muscle groups. The highest levels of DOMS were reported in the quadriceps and the upper back. Such high levels were unexpected in the upper back. However, it appears that this area involves a balancing/ braking action during downhill running. Most soreness peaked at $24 \mathrm{~h}$ and then began to dissipate. However, DOMS remained elevated in the tibialis anterior and gastrocnemius through $72 \mathrm{~h}$.

In conclusion, a somewhat puzzling aspect of the comparisons between this study involving low-intensity, highrepetition aerobic-like eccentrics versus studies involving high intensity, low repetition resistance-like eccentrics, is that certain aspects of the repeated bout effect are similar (CK was significantly reduced, although less pronounced elevations were seen in this study; DOMS was also significantly reduced after run 2), while other aspects of this response differ (different responses in circulating numbers of neutrophils after run 2). It is suggested that future studies be more cognisant of the mode of eccentrics used when attempting to interpret the responses to tissue damage. 


\section{REFERENCES}

1. American College of Sports Medicine. Guidelines for Exercise Testing and Prescription. 6th ed. Baltimore, MD: Lippincott Williams and Wilkins, 2000 .

2. Armstrong RB, Garshnek V, Schwane JA. Muscle inflammation: response to eccentric exercise. Med Sci Sports Exerc 1980; 12: S94-5.

3. Byrnes WC, Clarkson PM, White JS, Hseih SS, Frykman PN, Maughan RJ. Delayed onset muscle soreness following repeated bouts of downhill running. J Appl Physiol 1985; 59: 710-15.

4. Cleak MJ, Eston RG. Muscle soreness, swelling, stiffness and strength loss after intense eccentric exercise. Br J Sport Med 1992; 26: 267-72.

5. Eston RG, Lemmey AB, Mchugh P, Byrnes C, Walsh SE. Effect of stride length on symptoms of exercise-induced muscle damage during a repeated bout of downhill running. Scand J Med Sci Sports 2000; 10: 199-204.

6. Eston RG, Mickleborough J, Baltzopoulos V. Eccentric activation and muscle damage: biomechanical and physiological considerations during downhill running. Br J Sport Med 1995; 29: 89-94.

7. Faulkner JA, Brooks SV, Opiteck JA. Injury to skeletal muscle fibers during contractions: conditions of occurrence and prevention. Phys Ther 1993; 73: 911-21.

8. Hirose L, Nosaka K, Newton M, et al. Changes in inflammatory mediartors following eccentric exercise of the elbow flexors. Immunol Rev 2004; 10 : 75-90.

9. Lapointe $\mathrm{BM}$, Fremont $\mathrm{P}$, Cote $\mathrm{CH}$. Adaptations to lenghtening contractions is independent of voluntary muscle recruitment but relies on inflammation. Am J Physiol Regul Integer Comp Physiol 2002; 282: R323-29.

10. Lindstedt SI, Lastayo PC, Reich TE. When active muscles lengthen: Properties and consequences of eccentric contractions. New Physiol Sci 2001; 16 256-61.

11. Malm C, Nyberg $P$, Engstrom $M$, et al. Immunological changes in human skeletal muscle and blood after eccentric exercise and multiple biopsies. $J$ Physiol 2000; 529: 243-62.

12. Malm C, Sjodin B, Sjoberg B, et al. Leukocytes, cytokines, growth factors and hormones in human skeletal muscle and blood after uphill or downhill running. J Physiol 2004; 556: 983-1000.
13. McHugh M. Recent advances in the understanding of the repeated bout effect: a brief review. Scand J Med Sci Sports 2003; 13: 1-10.

14. Nosaka K, Newton M. Differences in the magnitude of muscle damage between maximal and submaximal eccentric loading. J Strength Cond Res 2002; 16: 202-8.

15. Nosaka K, Sakamoto K, Newton M, Sacco P. How long does the protective effect on eccentric exercise-induced muscle damage last? Med Sci Sports Exerc 2001; 33: 1490-5.

16. Peake JM, Nosaka K, Suzuki K. Characterization of inflammatory responses to eccentric exercise in humans. Exerc Immunol Rev 2005; 11: 64-85.

17. Pizza FX, Baylies H, Mitchell JB. Adaptations to eccentric exercise: neu trophils and E-selectin during early recovery. Can J Appl Physiol 2001 26: $245-53$.

18. Pizza FX, Davis BH, Henrickson SD, et al. Adaptation to eccentric exercise: effect on CD64 and CD11b/CD18 expression. J App/ Physiol 1996 80: 47-55

19. Pizza FX, Koh TJ, Mcgregor SJ, Brooks SV. Muscle inflammatory cells after passive stretches, isometric contractions, and lengthening contractions. J Appl Physiol 2001; 92: 1873-78.

20. Schwane JA, Armstrong RB. Effect of training on skeletal muscle injury from downhill running in rats. J Appl Physiol 1983; 55: 969-75.

21. Smith LL, Bond JA, Holbert D, et al. Differential white cell count after two bouts of downhill running. Int J Sports Med 1998; 19: 432-37.

22. Smith LL, Fulmer MG, Holbert D, et al. The impact of a repeated bout of eccentric exercise on muscular strength, muscle soreness, and creatine kinase. Br J Sports Med 1994; 28: 267-71.

23. Van der Meulen JH, Kuipers H, Drukker J. Relationship between exerciseinduced muscle damage and enzyme release in rats. J Appl Physiol 1991; 71: 999-1004

24. Warren GI, Lowe DA, Armstrong RB. Measurement tools used in the study of eccentric contraction-induced injury. Sports Med 1999; 27: 43-59. 